\title{
Cutoff Values of Plasma D-Dimer Level in Patients with Diagnosis of the Venous Thromboembolism after Elective Spinal Surgery
}

\author{
Saeed Hamidi ${ }^{1}$, Mahdieh Riazi ${ }^{2}$ \\ ${ }^{1}$ Division of Neurosurgery, Department of Surgery, Bou-Ali Medical Educational \& Clinical Center, \\ Tehran Medical Branch, Islamic Azad University, Tehran, Iran \\ ${ }^{2}$ Medical Science Research Center, Tehran Medical Branch, Islamic Azad University, Tehran, Iran
}

\begin{abstract}
Study Design: A prospective clinical study.
Purpose: Our objective in the present study was twofold. First, we sought to evaluate the relationship between postoperative venous thromboembolism (VTE) development and concentration of D-dimer to determine the cutoff value in patients who underwent elective spinal surgery. Second, we identified the predictive risk factors for postoperative VTE.

Overview of Literature: VTE affects the general health of patients and may even cause death. Since the complications of VTE are difficult to predict, the safest and most cost effective diagnostic method should be used in order to confirm a suspected VTE event after spinal surgery.

Methods: This study was performed on 97 patients who underwent elective spinal surgery. The D-dimer assay was carried out on the day before surgery, and on days 1, 3, and 10 following surgery. VTE occurrence and D-dimer levels were compared between the VTE and the control groups.

Results: Four patients $(4.1 \%)$ were diagnosed with deep vein thrombosis and one patient $(1 \%)$ was diagnosed with a pulmonary embolism. The optimum D-dimer cutoff value on day 3 following surgery in the VTE group was determined to be more than $2.1 \mu \mathrm{g} / \mathrm{mL}$ with a sensitivity of $100 \%$ and a specificity of $80.7 \%$. Moreover, the duration of postoperative recumbency was a significant risk factor for the development of VTE in this study.

Conclusions: We have demonstrated that postoperative D-dimer measurements in patients who underwent elective spinal surgery can provide a complementary diagnostic screening for VTE during the first week after surgery.
\end{abstract}

Keywords: D-dimer; Venous thromboembolism; Risk factors; Spine; Surgery

\section{Introduction}

Approximately 500,000 cases of venous thromboembolism (VTE) are reported annually in the United States. Deep vein thrombosis (DVT) and pulmonary embolism (PE) are two clinical manifestations of VTE. In fact,
VTE is essentially a massive PE, causing death in $70 \%$ within the first hour following the onset of symptoms [1-3]. VTE remains a significant complication after spinal surgery and according to various reports, the incidence of symptomatic VTE in patients who underwent spinal surgery has ranged from $0.3 \%-31 \%[4]$. Moreover, the

Received Jul 22, 2014; Revised Sep 9, 2014; Accepted Sep 9, 2014

Corresponding author: Saeed Hamidi

Division of Neurosurgery, Department of Surgery, Bou-Ali Medical Educational \& Clinical Center,

Tehran Medical Branch, Islamic Azad University, Tehran Medical Branch, Tehran, Iran

Tel: +98-21-88-56-3020,Fax: +98-21-88-57-1081,E-mail: hamidi_ns@yahoo.com 
incidence of VTE complications in patients who underwent major elective spinal surgery has been reported to be $8.3 \%-15.5 \%$ [5-8].

Since the complications arising solely from VTE cannot be diagnosed reliably by merely undergoing a clinical examination [9], in order to confirm a suspected VTE event after spinal surgery, the most cost effective and safest diagnostic method should be used. Although D-dimer levels in plasma have been shown to increase postoperatively [10], an optimum concentration level signifying a lower risk may fill the clinical role, ruling out VTE [11]. Even though the diagnostic significance of the D-dimer test is well-known for predicting VTE symptoms, the role of high D-dimer levels in the postoperative state following spinal surgery has been controversial and its cutoff level for predicting the risk of developing VTE still remains debatable. Furthermore, evidence based on previous studies indicate that postoperative D-dimer tests may not be accurate in sensitivity and specificity $[7,8,10,12-14]$.

The aim of the present study was to investigate the relationship between postoperative venous thromboembolic complications and the concentration of D-dimer to determine a cutoff value in patients who had undergone elective spinal surgery. Moreover, we sought to identify risk factors associated with VTE after spinal surgery.

\section{Materials and Methods}

A prospective study was carried out involving patients who underwent elective spinal surgery between 2011 and 2013 at the Islamic Azad University. The exclusion criteria were less than 18 years old, a history of venous thromboembolic complications prior to surgery, leukemia, sicklecell anemia, hepatic impairment, renal insufficiency, acute infections, active tuberculosis, pregnancy, receiving anticoagulation medication for over 48 hours prior to surgery, presence of a spinal cord injury, and having solid tumors. Patients were also excluded if their D-dimer or imaging results were incomplete. The study was approved by the institutional ethical committee. Written informed consent was acquired from each patient. Overall, thirteen patients were excluded from the study. Out of the ninetyseven remaining patients ( 45 male), the mean age ( \pm standard deviation, $\mathrm{SD}$ ) was $55.3 \pm 12.8$ years (ranging from 23 to 86 years), the mean body mass index (BMI) $( \pm \mathrm{SD})$ was $26.8 \pm 3.67 \mathrm{~kg} / \mathrm{m}^{2}$, and $21.9 \%$ of the subject population was obese $\left(\mathrm{BMI} \geq 30 \mathrm{~kg} / \mathrm{m}^{2}\right)$. The diagnoses at admission in the study population are shown in Fig. 1. All surgeries were performed under general anesthesia. The operative level of surgery, duration of surgery, intraoperative transfusions, and blood loss were all recorded immediately after surgery. The postoperative blood drainage was recorded as well.

Venous blood samples were collected to measure D-dimer levels using the ELISA method (VIDAS, bioMérieux, France) before and 1, 3, and 10 days after surgery. All patients received postoperative elastic stockings prophylaxis. Physical evaluation (with the Wells score system) related to new acute VTE symptoms was performed the day before the operation, during hospitalization, and within two weeks after surgery by the same examiner; additionally, bilateral venous Doppler ultrasonography was performed for all patients after surgery during hospitalization by the same radiologist. The computed tomography (CT) angiography was also used to evaluate suspected PE events after surgery. Moreover, patients with a D-dimer level higher than $0.5 \mu \mathrm{g} / \mathrm{mL}$ were followed up for the development of VTE postoperatively.

We performed stepwise logistic regression to identify factors that were predictive of VTE among seven independent variables (age, sex, BMI, operative time, operative level, bleeding volume including intra- and postoperative blood loss, and duration of postoperative recumbency). To assess the D-dimer cutoff point, a receiver operating characteristic (ROC) analysis was con-

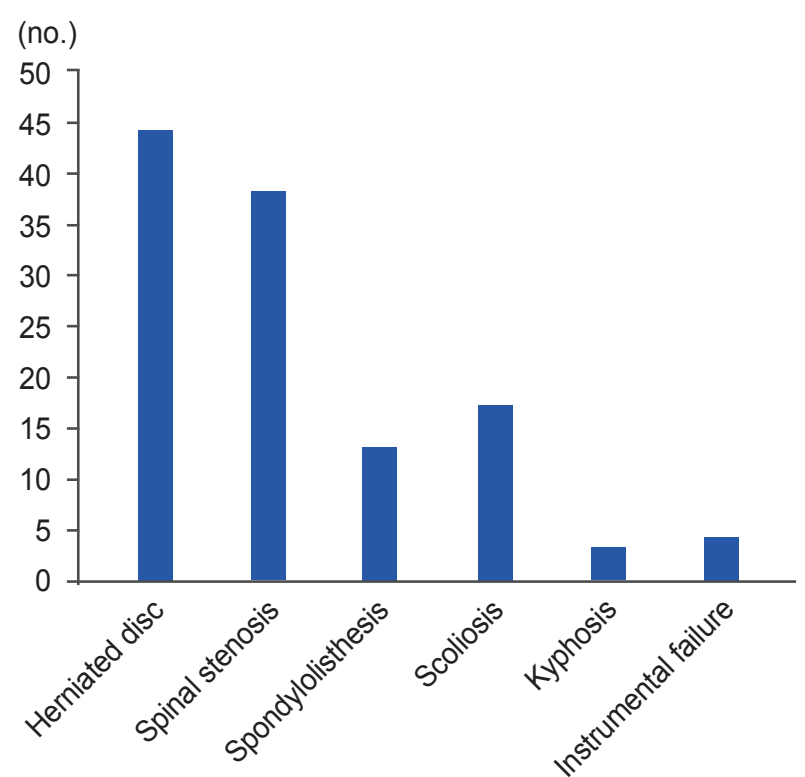

Fig. 1. Diagnosis of 97 patients. Some patients were diagnosed with more than one complication. 
Table 1. Demographic data of 97 patients

\begin{tabular}{lccc}
\hline Characteristic & VTE positive $(\mathrm{n}=5)$ & VTE negative $(\mathrm{n}=92)$ & $p$-value \\
\hline Sex (male:female=45:52) & $4: 1$ & $41: 51$ & 0.180 \\
\hline Age $(\mathrm{yr})$ & $60.0 \pm 13.6$ & $55.0 \pm 12.8$ & 0.323 \\
\hline Body mass index $\left(\mathrm{kg} / \mathrm{m}^{2}\right)$ & $26.4 \pm 2.0$ & $26.9 \pm 3.7$ & 0.862 \\
\hline Operative time $(\mathrm{min})$ & $226.0 \pm 55.4$ & $190.0 \pm 65.9$ & 0.160 \\
\hline Bleeding volume $(\mathrm{mL})^{\text {a) }}$ & $801.0 \pm 315.8$ & $709.2 \pm 305.7$ & 0.310 \\
\hline Duration of postoperative recumbency $(\mathrm{hr})$ & $46.4 \pm 17.3$ & $21.2 \pm 9.2$ & $0.001^{\mathrm{b})}$ \\
\hline Operative level & & & 10 \\
\hline Cervical & 0 & 4 & $>0.99$ \\
\hline Thoracic & 0 & 74 & $>0.99$ \\
\hline Lumbar & 5 & 3 & 0.583 \\
\hline More one level & 0 & $>0.99$ \\
\hline
\end{tabular}

Values are presented as mean \pm standard deviation.

Fisher's exact test was used with regard to sex and operative level. Mann-Whitney U test was used for other data. $p$-values are 2-tailed.

VTE, venous thromboembolism.

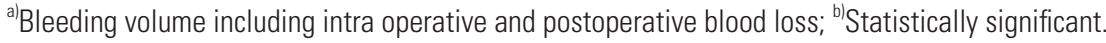

Table 2. Stepwise logistic regression for risk factors

\begin{tabular}{|c|c|c|}
\hline Risk factors (covariate) & $p$-value & Odd ratio $(95 \% \mathrm{CI})$ \\
\hline Sex & 0.564 & Not significant \\
\hline Age & 0.167 & Not significant \\
\hline Body mass index & 0.182 & Not significant \\
\hline Operative |evel|a) & 0.838 & Not significant \\
\hline Operative time & 0.204 & Not significant \\
\hline Duration of postoperative recumbancy & 0.003 & $1.169(1.053-1.298)$ \\
\hline Bleeding volume $^{b /}$ & 0.693 & Not significant \\
\hline
\end{tabular}

Values are presented as $95 \%$ confidence interval (CI).

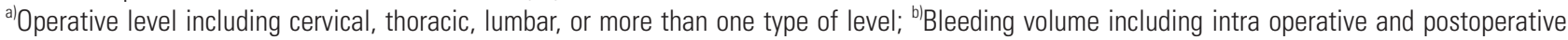
blood loss.

ducted. We performed Fisher's Exact and Mann-Whitney $\mathrm{U}$ tests to compare the VTE-positive and VTE-negative groups. SPSS ver. 21 (IBM Co., Armonk, NY, USA) was used, and $p$-values less than 0.05 were considered statistically significant.

\section{Results}

Five patients (5.1\%) had postoperative thrombotic complications. One patient (1.0\%) developed PE without prior clinical signs of DVT. Out of four cases diagnosed with DVT (4.1\%), three were asymptomatic. Isolated distal DVT was identified in three (3.0\%) patients, and we detected combined proximal and distal DVT in one patient (1.0\%).

The mean age ( 60 years vs. 55 years, VTE vs. non-VTE), BMI $\left(26.4 \mathrm{~kg} / \mathrm{m}^{2}\right.$ vs. $\left.26.9 \mathrm{~kg} / \mathrm{m}^{2}\right)$, operative time (226 min vs. $190 \mathrm{~min})$, bleeding volume ( $801 \mathrm{~mL}$ vs. $709.2 \mathrm{~mL})$, and operative level did not differ significantly between the VTE and the non-VTE groups, while all thromboembolic complications were found in patients who underwent spinal surgery. The recumbent duration after surgery was significantly higher in the VTE group compared to the non-VTE group, as depicted in Table 1. The mean recumbent time was 46.4 hours in patients with VTE. Logistic regression also showed that duration of postoperative recumbency was a significant independent risk factor for developing VTE (Table 2). 
The changes in values of the $\mathrm{D}$-dimer levels preoperatively and on postoperative day 1,3, and 10 are presented in Fig. 2. As expected, the concentration of D-dimer was found to increase postoperatively. Among all measured levels, the mean value of $\mathrm{D}$-dimer peaked on postoperative day 3 for the VTE group. However, in the non-VTE group, the mean value of $\mathrm{D}$-dimer peaked on the first day after surgery. Statistically, there was no significant difference between the preoperative $\mathrm{D}$-dimer levels between the VTE and non-VTE groups $(0.68 \pm 0.47 \mu \mathrm{g} / \mathrm{mL}$ vs. $0.41 \pm 0.33 \mu \mathrm{g} / \mathrm{mL} ; p=0.169)$, but $\mathrm{D}$-dimer levels on the first $(3.79 \pm 1.48 \mu \mathrm{g} / \mathrm{mL}$ vs. $2.08 \pm 1.71 \mu \mathrm{g} / \mathrm{mL} ; p=0.025)$, third $(5.60 \pm 2.96 \mu \mathrm{g} / \mathrm{mL}$ vs. $1.52 \pm 1.35 \mu \mathrm{g} / \mathrm{mL} ; p=0.001)$, and tenth $(2.11 \pm 0.97 \mu \mathrm{g} / \mathrm{mL}$ vs. $1.02 \pm 1.10 \mu \mathrm{g} / \mathrm{mL}$; $p=0.028)$ day after operation were significantly higher in the VTE group compared to those without VTE.

The cutoff value with relevant sensitivity and specificity was predicted by ROC analysis. The area under the curve corresponding to the $\mathrm{D}$-dimer on the third day after surgery proved to be the best indicator for anticipating VTE symptoms (Fig. 3). The optimum cutoff concentration of $\mathrm{D}$-dimer on postoperative day 3 for diagnosing the onset of VTE symptoms was determined to be $2.113 \mu \mathrm{g} / \mathrm{mL}$. At this level, the sensitivity and specificity were $100 \%$ and $80.7 \%$, respectively, with a positive predictive value (PPV) of $31.2 \%$ and negative predictive value (NPV) of $100 \%$.

\section{Discussion}

Since the onset of VTE is difficult to predict using individual clinical examinations [9], screening methods are important tools for determining whether VTE complications are occurring [4]. However, prior instrumental diagnoses have been considered an unsuitable tool for routine

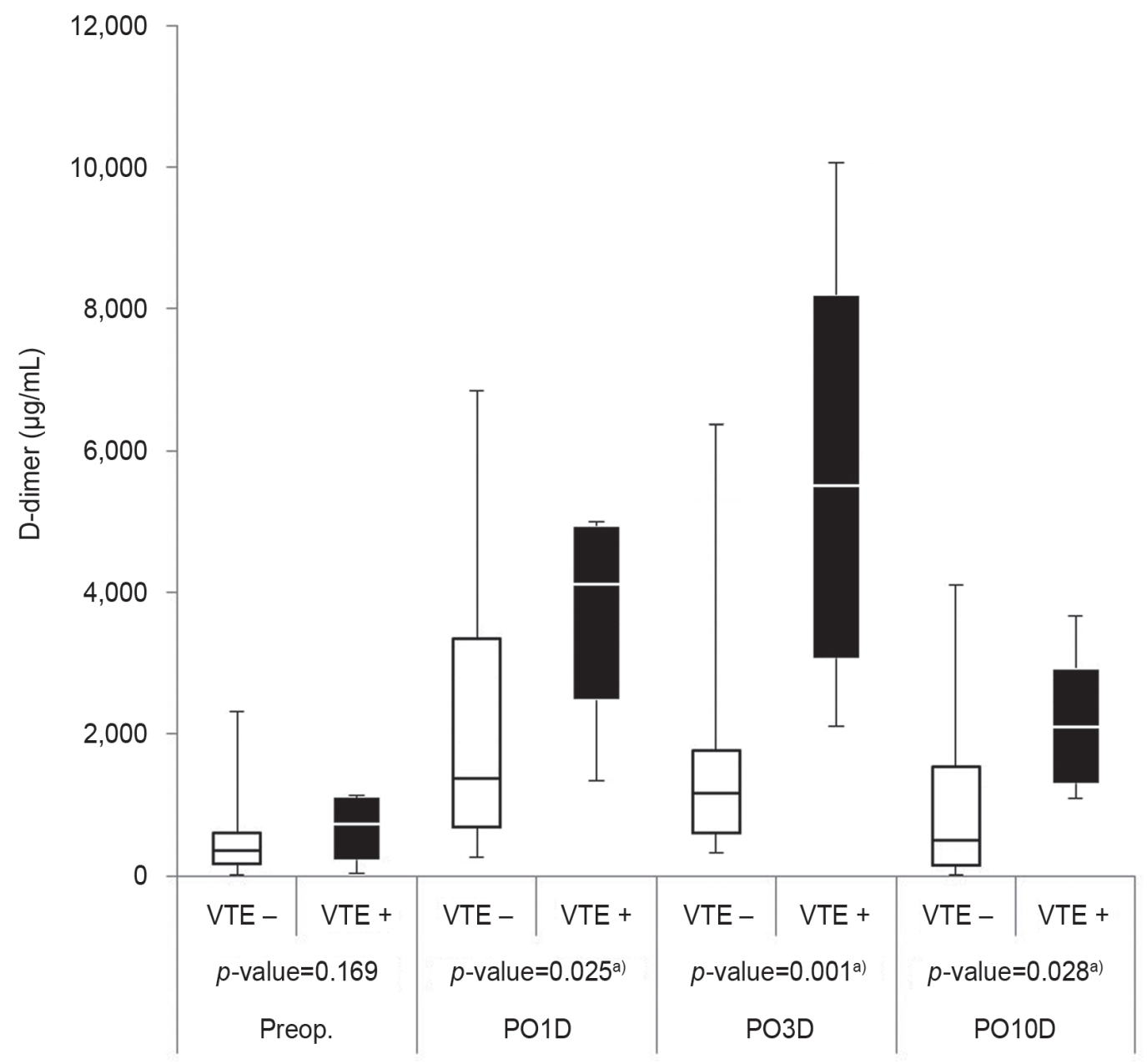

Fig. 2. The changes in values of D-dimer, preoperatively (Preop.) and on postoperative days 1 (P01D), 3 (P03D), and 10 (P010D). Mann-Whitney U test was used. VTE, venous thromboembolism. ${ }^{\text {al }}$ Statistically significant. 
evaluation of asymptomatic VTE complications due to high costs, complications, and low accuracy [15]. In addition, D-dimer levels in the plasma below the standard threshold level of $0.5 \mu \mathrm{g} / \mathrm{mL}$ can serve as a cost effective screening strategy to eliminate VTE [14]. However, the concentration of D-dimer has been shown to increase after surgery [10]; hence, the application of a D-dimer test exclusively to negate postoperative VTE complications is limited. In our study, D-dimer levels increased after surgery, and postoperative $\mathrm{D}$-dimer levels were significantly higher in the VTE group $(p<0.05)$. These results are in agreement with previous studies that have associated elevated levels of D-dimer with the development of VTE after spinal surgery $[5,7,8]$. In a previous study, Dindo et al. [14] reported that the optimal time period for the D-dimer tests during the postoperative period depended on the invasiveness, type, and duration of the operation. It has been reported that, for the diagnosis of VTE after spinal surgery, this time frame should be within the first week of the postoperative period $[5,7,8]$.

In an earlier study comprising 72 patients who underwent spinal surgery, Yoshioka et al. [8] evaluated the overall sensitivity to be $83.3 \%$ with s NPV of $98.0 \%$ when the D-dimer cutoff level was assigned a value of $6.5 \mu \mathrm{g} /$ $\mathrm{mL}$ on postoperative day 7 ; hence, they concluded in view of their poor findings, that the D-dimer level was unsuitable for use as an early diagnostic to predict VTE. In contrast, Yoshiiwa et al. [7] set the D-dimer level cutoff point at $10 \mu \mathrm{g} / \mathrm{mL}$ in their study, and reported that the sensitivity and specificity of D-dimer measurements on day 4 after spinal surgery were $100 \%$ and $95.1 \%$, respectively. As mentioned earlier, we measured D-dimer levels on days 1, 3, and 10 after surgery. Since any screening method to negate VTE should demonstrate high sensitivity and NPV, our results from the ROC analysis showed that $\mathrm{D}$-dimer levels on the third day after surgery would be good indicators for the prediction of VTE events. Based on our assay results, a threshold value of $2.113 \mu \mathrm{g} /$ $\mathrm{mL}$ on postoperative day 3 provided $100 \%$ sensitivity and a NPV with a specificity of $80.7 \%$ and a PPV of $31.2 \%$. Compared to previous reports, the D-dimer cutoff point derived from our study was relatively low. However, one study included patients having solid tumors [8], which may be the reason for their high levels of D-dimer. In an-

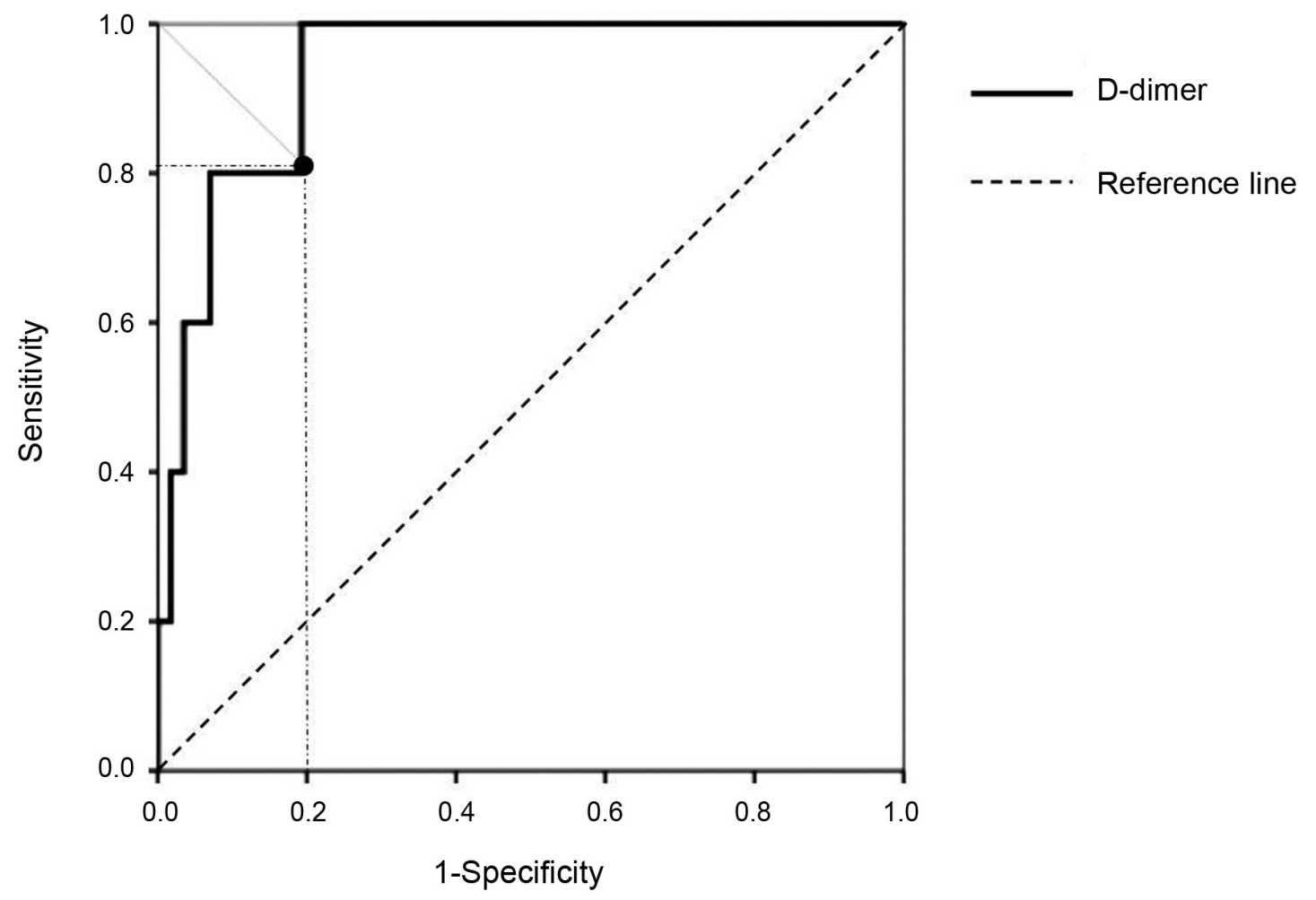

Fig. 3. Receiver operating characteristic analysis of D-dimer on the postoperative day 3; area under the curve $=0.937, p$ value=0.001; asymptomatic 95\% confidence interval, 0.861-1.000. 
other study, the imaging method for screening for DVT and $\mathrm{PE}$ was not performed at $\mathrm{D}$-dimer levels less than 10 $\mu \mathrm{g} / \mathrm{mL}$, so cases of asymptomatic VTE might have been missed [7]. Since DVT with subsequent pulmonary emboli is a likely complication after spinal surgery, it would be better to use a more sensitive screening method for the detection of symptomatic and asymptomatic VTE. This is why our study has a different sensitivity in comparison to other studies. Moreover, a similar conclusion was derived from a study that examined D-dimer concentrations one week after spinal surgery. Patients who had D-dimer levels $\geq 10 \mu \mathrm{g} / \mathrm{mL}$, did not suffer from VTE complications; on the other hand, patients who developed asymptomatic VTE, had mean D-dimer levels below $5.7 \mu \mathrm{g} / \mathrm{mL}$ [5]. Thus, choice of the cutoff value depended on the methods employed and populations studied.

From this study, we have obtained a convincing threshold value for D-dimer levels in the plasma. Out of the five VTE diagnosed cases (5.1\%), three were asymptomatic, and therefore this value can be used as a reliable tool for asymptomatic DVT screening. Screening for asymptomatic DVT can prevent PE. These results are in agreement with previous research that evaluated the incidence of asymptomatic thromboembolic events after spinal surgery $[5,7,8,16]$.

Statistically no significant differences related to sex, mean age, BMI, operative time, bleeding volume, and operative level were observed; however, all thromboembolic complications were found in patients who had undergone lumbar surgery in the prone position with a posterior approach. These results agree with the findings from other studies that have linked postoperative VTE complications with intraoperative position during surgery [6]. Indeed, our statistical validation can be attributed to the sample size of ninety-seven patients in the present study. VTE should be diagnosed within 14 days after surgery $[5,6,8]$. So, we sought to investigate changes of $\mathrm{D}$-dimer levels during this time frame. According to our hospitals protocol, patients who undergo elective spinal surgery and do not have any complications should be discharged on the third day after surgery and have their first postop visit seven days later. Therefore in order to be cost effective and efficient, we measured the level of D-dimer on the postop 1, 3, and 10 days. There are several other limitations associated with our study. For instance, CT angiography was not performed to detect $\mathrm{PE}$ in each patient because of potential side effects and medical costs. More- over, most of the patients who underwent elective surgery were suffering from a lumbar disease. Also, our study included patients and procedures confined to a particular region.

\section{Conclusions}

In summary, the routine use of a threshold value of $\mathrm{D}$ dimer levels at $2.1 \mu \mathrm{g} / \mathrm{mL}$ on the third postoperative day in patients who opted to undergo elective spinal surgery, warrants complementary diagnostic screening. VTE develops within the first week after surgery, so an early diagnosis will permit treatment to be initiated as early as possible. Since this test appears to be more effective than clinical examinations alone, it can be used as a screening test for the early detection of VTE. We have also demonstrated that the recumbent duration after surgery is a significant risk factor for the development of DVT and PE. Overall, further studies are needed to investigate the association between D-dimer threshold levels and other risk factors associated with spinal surgery.

\section{Conflict of Interest}

No potential conflict of interest relevant to this article was reported.

\section{References}

1. Agnelli G, Sonaglia F. Prevention of venous thromboembolism. Thromb Res 2000;97:V49-62.

2. Muntz JE. Deep vein thrombosis and pulmonary embolism in the perioperative patient. Am J Manag Care 2000;6(20 Suppl):S1045-52.

3. Hamilton MG, Hull RD, Pineo GF. Venous thromboembolism in neurosurgery and neurology patients: a review. Neurosurgery 1994;34:280-96.

4. Glotzbecker MP, Bono CM, Wood KB, Harris MB. Thromboembolic disease in spinal surgery: a systematic review. Spine (Phila Pa 1976) 2009;34:291-303.

5. Takahashi $\mathrm{H}$, Yokoyama $\mathrm{Y}$, Iida $\mathrm{Y}$, et al. Incidence of venous thromboembolism after spine surgery. J Orthop Sci 2012;17:114-7.

6. Oda T, Fuji T, Kato Y, Fujita S, Kanemitsu N. Deep venous thrombosis after posterior spinal surgery. Spine (Phila Pa 1976) 2000;25:2962-7.

7. Yoshiiwa T, Miyazaki M, Takita C, Itonaga I, Tsumu- 
ra H. Analysis of measured D-dimer levels for detection of deep venous thrombosis and pulmonary embolism after spinal surgery. J Spinal Disord Tech 2011;24:E35-9.

8. Yoshioka K, Kitajima I, Kabata T, et al. Venous thromboembolism after spine surgery: changes of the fibrin monomer complex and D-dimer level during the perioperative period. J Neurosurg Spine 2010;13:594-9.

9. Bono CM, Watters WC 3rd, Heggeness MH, et al. An evidence-based clinical guideline for the use of antithrombotic therapies in spine surgery. Spine J 2009;9:1046-51.

10. Levy G, Levy PY, Hessmann J, Monin P. Diagnosis of post-operative venous thrombosis using determination of plasma D-dimer. J Mal Vasc 1998;23:269-73.

11. Ljungqvist M, Soderberg M, Moritz P, Ahlgren A, Larfars G. Evaluation of Wells score and repeated Ddimer in diagnosing venous thromboembolism. Eur J Intern Med 2008;19:285-8.

12. Yukizawa Y, Inaba Y, Watanabe S, et al. Association between venous thromboembolism and plasma levels of both soluble fibrin and plasminogen-activator inhibitor 1 in 170 patients undergoing total hip arthroplasty. Acta Orthop 2012;83:14-21.

13. Sudo A, Wada H, Nobori T, et al. Cut-off values of Ddimer and soluble fibrin for prediction of deep vein thrombosis after orthopaedic surgery. Int J Hematol 2009;89:572-6.

14. Dindo D, Breitenstein S, Hahnloser D, et al. Kinetics of D-dimer after general surgery. Blood Coagul Fibrinolysis 2009;20:347-52.

15. Davidson HC, Mazzu D, Gage BF, Jeffrey RB. Screening for deep venous thrombosis in asymptomatic postoperative orthopedic patients using color Doppler sonography: analysis of prevalence and risk factors. AJR Am J Roentgenol 1996;166:659-62.

16. Strom RG, Frempong-Boadu AK. Low-molecularweight heparin prophylaxis 24 to 36 hours after degenerative spine surgery: risk of hemorrhage and venous thromboembolism. Spine (Phila Pa 1976) 2013;38:E1498-502. 